\title{
High frequency content in nonlinear soil response: A numerical artifact or a reality?
}

\author{
Veeraraghavan, S., Spears, R. E., \\ Coleman, J. L. \\ January 2019
}

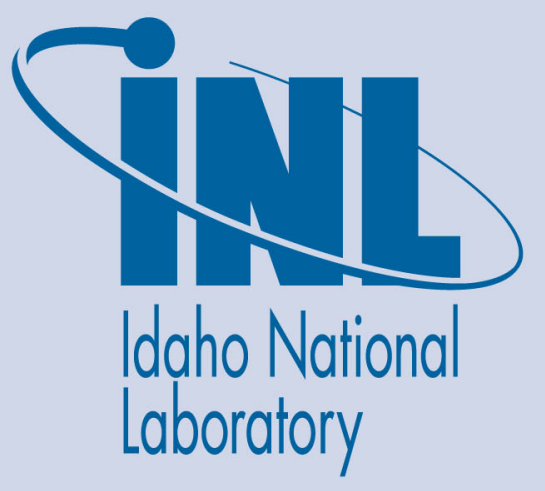

The INL is a U.S. Department of Energy National Laboratory operated by Battelle Energy Alliance 


\section{High frequency content in nonlinear soil response: A numerical artifact or a reality?}

Veeraraghavan, S., Spears, R. E., Coleman, J. L.

January 2019

Idaho National Laboratory Idaho Falls, Idaho 83415

http://www.inl.gov

Prepared for the

U.S. Department of Energy

Under DOE Idaho Operations Office

Contract DE-AC07-05ID14517 
High frequency content in nonlinear soil response: A numerical artifact or a reality?

Swetha Veeraraghavan” swetha.veeraraghavan@inl.gov, Robert E. Spears, Justin L. Coleman

Idaho National Laboratory, 2525 N. Fremont Ave, Idaho Falls, ID 83415, USA

${ }^{*}$ Corresponding author.

\section{Abstract}

This article probes the reasons for the presence of high frequency components, which are not a part of the input, in the output of nonlinear site response analysis. The nonlinear site response analysis of a single soil element, under a single frequency sinusoidal displacement, is conducted to demonstrate the presence of frequencies higher than the input frequency in the soil element's shear stress and the free surface acceleration. The parameters of this one element problem are chosen carefully to minimize numerical errors. The shape of the stress-strain hysteresis loop is found to be the main cause for the presence of these higher frequencies. A quasi-static problem similar to the direct shear test is then considered to analyze the effect of different hysteresis shapes. Through this analysis, it is numerically and analytically shown that any deviation of the stress-strain hysteresis shape from a perfect ellipse results in output frequencies higher than the input frequency and the number of higher frequencies produced depends on whether the hysteresis shape is smooth or pointed.

\section{Highlights}

- Causes for high frequency content in nonlinear soil response is explored.

- Insufficient spatial and temporal numerical discretization is a known cause.

- Numerical errors are minimized and an analytical response of the soil is obtained.

- Strong connection between stress-strain shape and high frequency is demonstrated.

- Any deviations from an elliptical hysteresis shape cause high frequency overtones.

\section{Keywords}

Nonlinear soil response; Site response analysis; High frequency noise

\section{Introduction}

Nonlinear stress-strain behavior of soils in a controlled laboratory setting have been calculated in numerous experimental studies conducted on a wide range of soils subjected to varying strain levels [29]. Additionally, field observations of recorded earthquake ground motions show that peak accelerations are well into the nonlinear range during shaking $[4,9,10,18]$. These 
experimental and field observations led to development of numerical models that approximate the nonlinear behavior of soils.

These numerical models can be divided into two major categories: (a) equivalent linear models and (b) fully nonlinear models. A brief description of these numerical models is provided in Section 1.1. Nonlinear soil models produce high frequency response due to multiple factors: the shape of the hysteresis loop, the pointed end of the hysteresis loop, and improper time/space discretization or other similar numerical discretization or integration issues. High frequency response could also be due to the input motion having large amplitudes at these frequencies. However, this article is focused on numerically and analytically describing the high frequency soil response due to the shape of the hysteresis loop and the pointed end of the hysteresis loop.

\subsection{Background on nonlinear soil models}

As mentioned earlier, the two major categories of numerical soil models are equivalent linear and direct nonlinear methods. In the equivalent linear method [14,28], the soil material properties such as shear modulus and damping ratio are modified iteratively based on the effective strain experienced by the soil. Starting with the corresponding low-strain values for the shear modulus and damping ratio, a linear analysis is performed and a single strain measure called the "effective strain" is calculated from the strain time history of the soil. Using this effective strain and the experimentally obtained modulus reduction and damping curves, the shear modulus and damping ratio for the next linear analysis are calculated, and this process is continued until the soil properties between two subsequent iterations converge (within a pre-defined tolerance). The key point to note here is that the nonlinear response of the soil is approximated using a series of linear analyses. Since a linear analysis is at the core of this method, the frequency content of the input and the output are similar, i.e., if the base of the soil column is shaken by applying a sinusoidal displacement of a single frequency, the displacement calculated at the top of the soil column will have the same single frequency as the input. This method has been implemented in 1D site response analysis tools such as SHAKE [28]. Modified versions of this method are also available where the shear modulus and damping ratio vary with both the frequency of excitation and the effective shear strain $[1,33]$.

In the direct nonlinear site response analysis, the soil properties are updated at each time step based on the current strain level experienced by the soil. Therefore, the nonlinear soil model approximates the soil stiffness and energy dissipated at each time step. To model the nonlinear response of the soil in 1D, the stress-strain backbone bone curve used to numerically represent the soil, and the hysteresis rules, which dictate the shape of the hysteresis loops under cyclic excitation, are required. Many researchers have developed empirical expressions that approximate the experimentally observed backbone curve [8,12,16,20,25]. Extended Masing rules are the most commonly used hysteresis rules to model the cyclic behavior of soils [19]. DEEPSOIL [13], DESRA [16], DMOD [21] and Tess [24] are some of the commonly used 1D site response analysis tools that use these backbone curves and hysteresis rules to model the nonlinear response of the soil. Some finite element tools also have a material model (e.g., Mat Hysteretic soil in LS-DYNA [17], user hysteretic soil in ABAQUS [31], I-Soil in MASTODON [6]) that uses the information from this $1 \mathrm{D}$ stress-strain curve to model the $3 \mathrm{D}$ nonlinear behavior of the soil. These material models are based on the distributed element model 
(developed by Iwan [15] and later adapted by Chiang and Beck [3]), in which the soil element is modeled as a set of elastic-perfectly plastic springs. The total stress of the soil element is the sum of stresses from each of the individual springs. J2 or von Mises plasticity [32] is used to model the 3D behavior of each elastic-perfectly plastic spring. This seemingly simple material model automatically satisfies Masing hysteresis rules. Advanced constitutive models which incorporate more sophisticated yield surfaces, hardening laws, and flow rules have also been developed $[7,22,23,26,27]$. Benchmarking of the commonly used methods for direct nonlinear site response analysis is presented in Stewart et al. [30].

\subsection{Past research on high frequency nonlinear soil response}

Yu et al. [34] compared response spectra from linear and nonlinear site response analysis of soil columns under an impulse excitation and concluded that the soil response can be divided into three frequency bands (Fig. 1). In the lower frequency, the spectral amplitude remains unaffected by the material model. In the central band, the nonlinear soil model has lower spectral amplitude than the linear soil model as a result of the nonlinear hysteretic damping. However, in the high frequency band, the reverse is true, i.e., the nonlinear soil model has higher spectral amplitude than the linear soil model. Yu et al. [34] attributed this response amplification of nonlinear soils in higher frequencies to the sudden change in soil stiffness at reversals in the stress-strain curve. Other researchers have also observed these high frequency components in the comparisons between equivalent linear and direct nonlinear methods (e.g., Bolisetti et al. [2]).

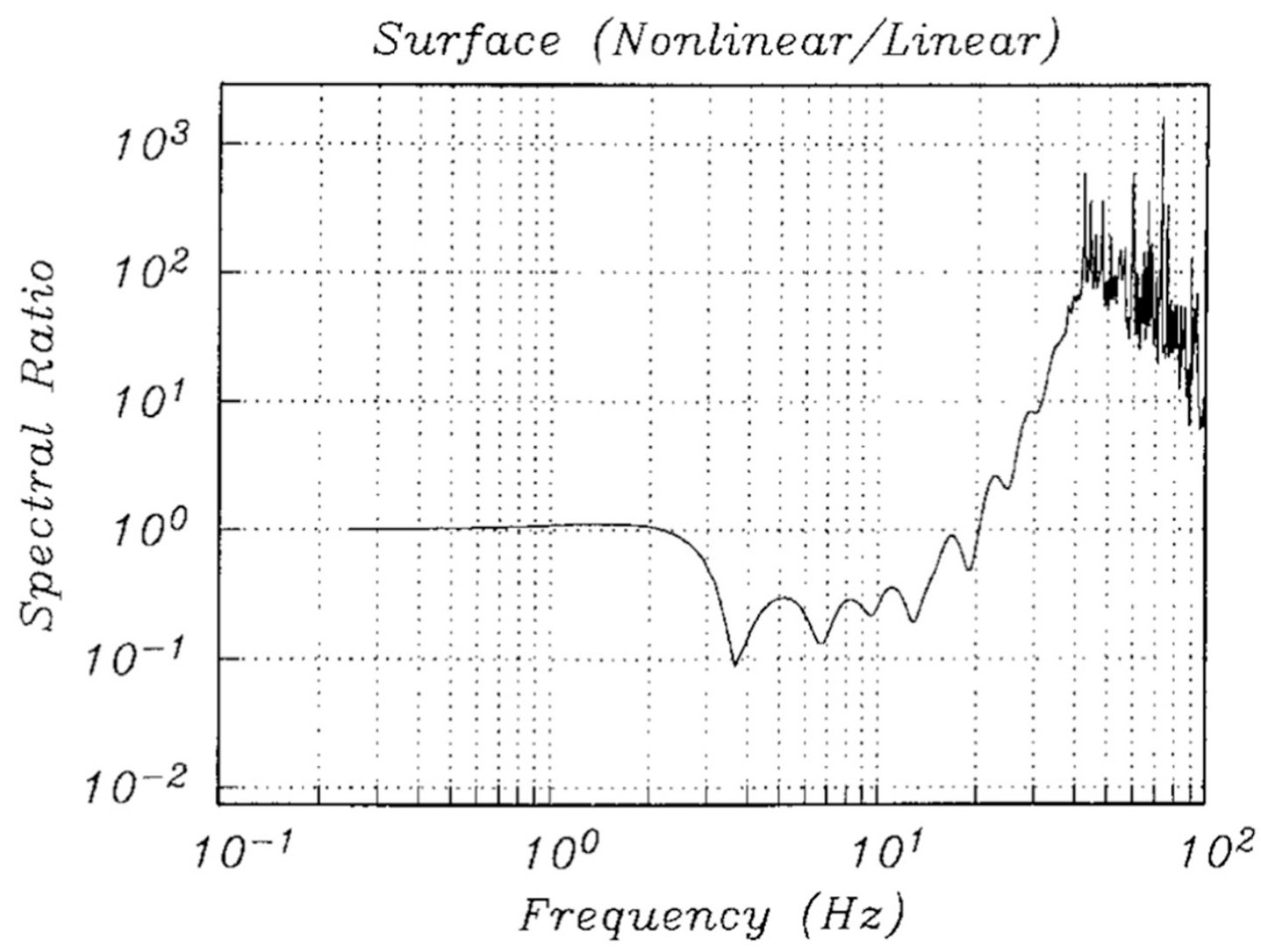


Fig. 1 Spectral ratio at the free surface calculated as nonlinear response divided by linear response for an input impulse pulse with peak acceleration of $0.5 \mathrm{~g}$ applied at the base of the soil column (reprinted from Yu et al. [34]).Fig. 1

Some of the other common causes for the presence of higher frequencies in time domain analysis are inadequate soil element size and time step increment size (Coleman et al. [5]). Considering that most of the machinery and equipment are sensitive to high frequency ground motion, it is important to investigate all the possible causes resulting in the high frequency nonlinear response of soils and to separate numerical noise from the actual soil response. In this article, nonlinear site response analysis of a single soil element is conducted under a single frequency sinusoidal excitation to track down all the possible causes for the presence of frequencies higher than the input frequency.

\section{Demonstration of high frequency nonlinear response}

A 125 feet (ft) tall soil element is considered for this study (Fig. 2). The base of this soil element is excited using a sinusoidal displacement with a frequency of $1.22 \mathrm{~Hz}$ and the top of the soil element is free. The maximum amplitude of the sinusoidal displacement is gradually increased from $0 \mathrm{ft}$ to $0.115 \mathrm{ft}$ over $2 \mathrm{~s}$ and then kept constant at $0.115 \mathrm{ft}$ for the remainder of the excitation, which extends till $51 \mathrm{~s}$, to ensure that the soil experiences a periodic sinusoidal displacement of a constant maximum amplitude after $2 \mathrm{~s}$. A truncated version of the applied displacement is shown in Fig. 2. The gradual increase in maximum amplitude of the sinusoidal displacement ensures that at $t=0$, the displacement, velocity and acceleration at the base of the soil column are zero. The density of the soil element is $118 \mathrm{lb} / \mathrm{ft}^{3}$ and the stress-strain behavior of this soil element is similar to that of alluvial soil (Fig. 3a). The element size (125 ft) and the time step size $(0.000625 \mathrm{~s})$ are chosen according to the criteria presented in Coleman et al. [5] so that high frequency numerical noise is avoided during the propagation of the wave. 

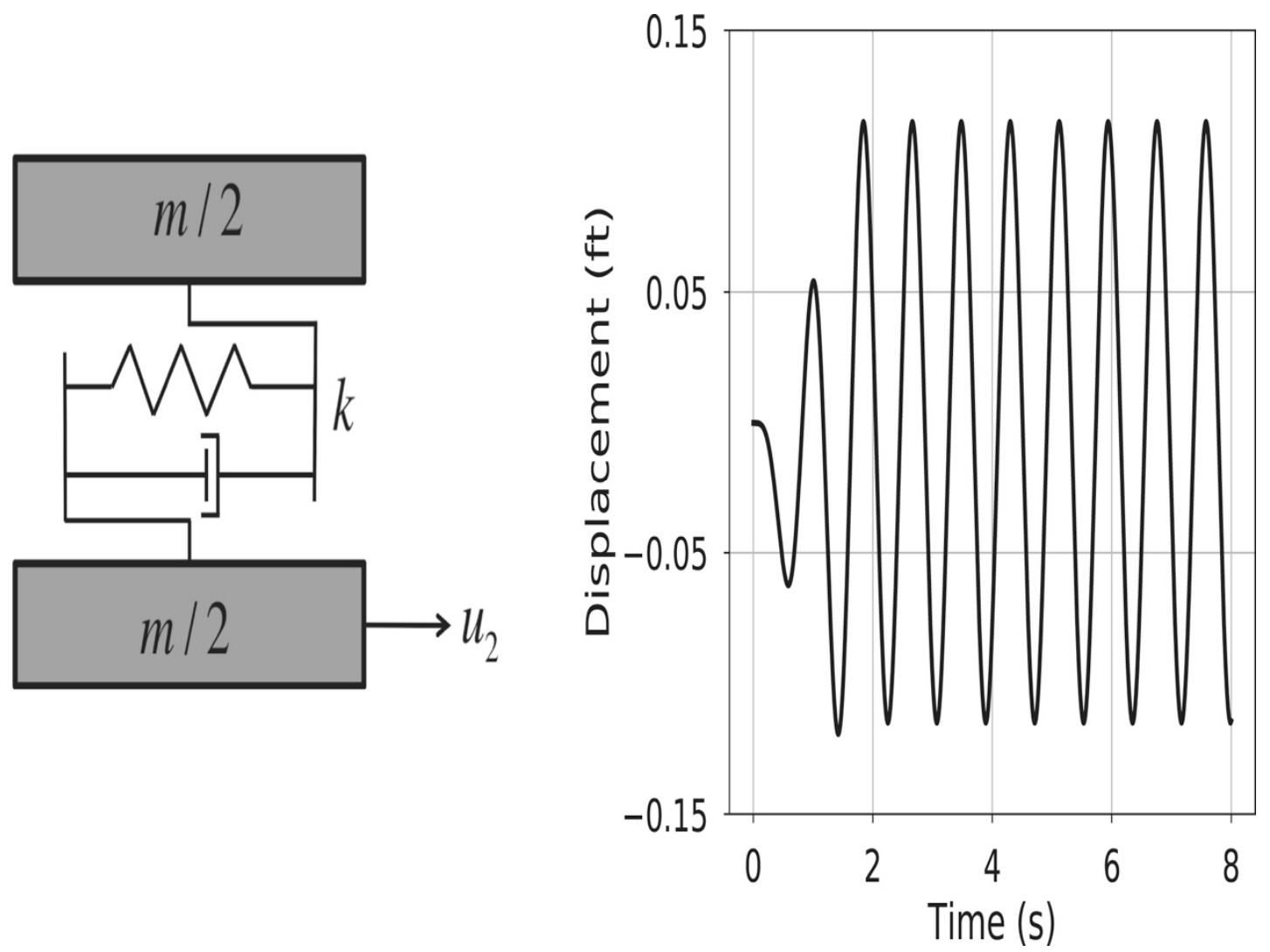

Fig. 2 One element soil model, represented using a spring mass damper system, with a sinusoidal displacement applied at the base. The top of the soil element is free.Fig. 2 

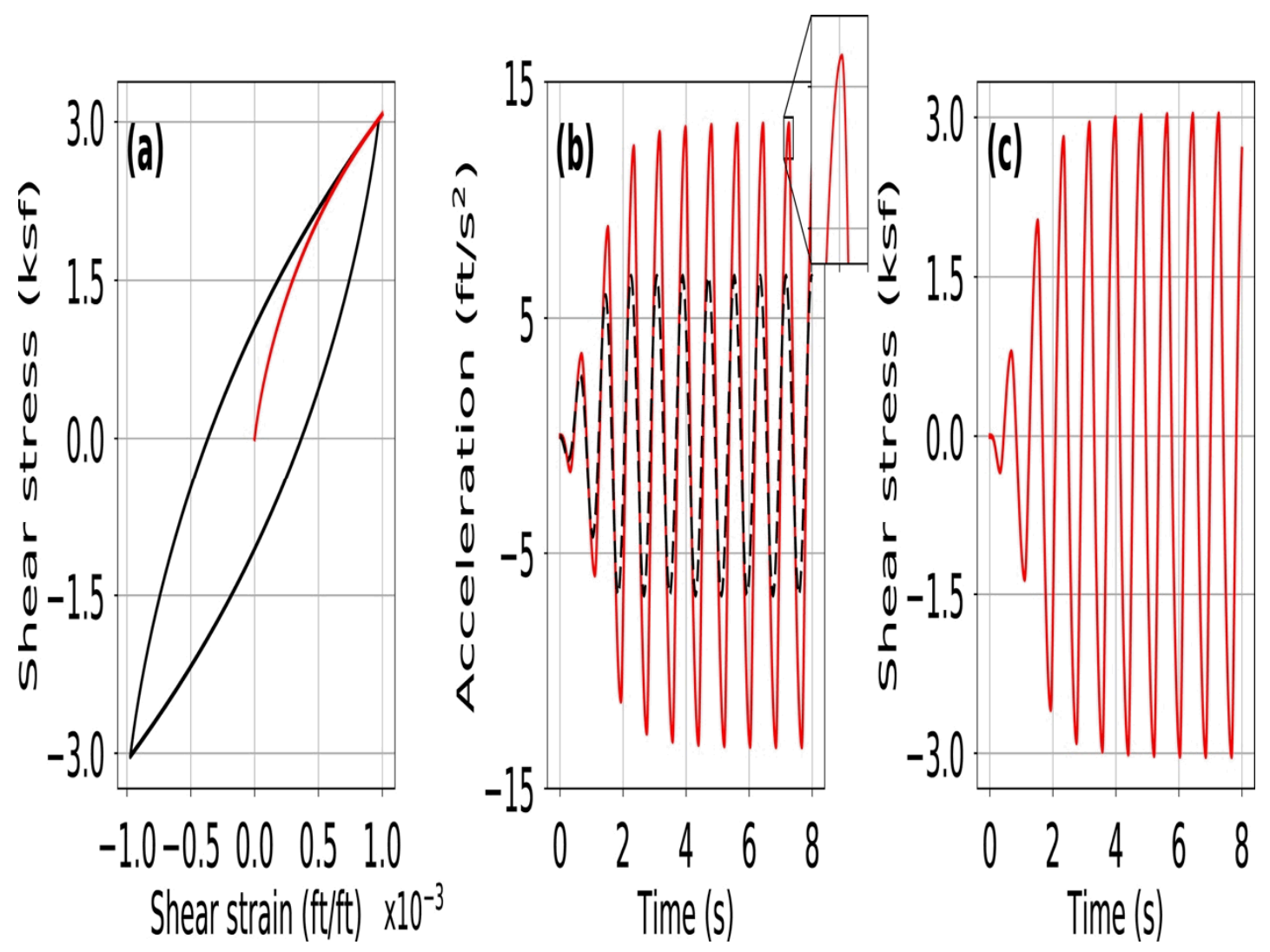

Fig. 3 (a) Shear stress vs shear strain backbone curve (red) and steady state hysteretic stressstrain behavior (black) for the nonlinear soil model. (b) Acceleration time history at the base (dashed black curve) and top (red curve) of the soil column. Zoomed inset shows the assymetry in the waveform to the left and right of the peak acceleration demonstrating the deviation from a perfect sinusoid. (c) Shear stress time history of the soil element.Fig. 3

This soil element is similar to a spring mass damper system (Fig. 2). The total mass of the element $(\mathrm{m})$ is split into two and the two halves are lumped into the node at the top and bottom of the soil element. A nonlinear spring-damper, the properties of which are given by the shear stress-shear strain curve in Fig. 3a, connect these two nodes. The governing equation for this 1D system is:

$$
\frac{1}{2} m u_{1}+f\left(u_{1}, u_{2}\right)=0
$$

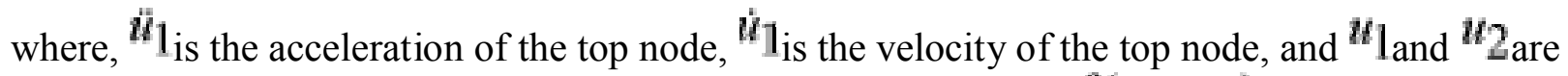
the displacements of the top and bottom nodes, respectively, and $f\left(u_{1}, u_{2}\right)_{\text {is }}$ the internal force generated by the nonlinear spring-damper system. This above equation along with time integration relations between acceleration, velocity and displacement are simultaneously solved to obtain ${ }^{\bar{u}_{1}}, \dot{u}_{1}{ }_{\text {and }}{ }^{u_{1}}$ as a function of time. Any time stepping algorithm such as Newmark-Beta time integration, forward Euler or Runge-Kutta methods could be used to solve the above 
nonlinear differential equation iteratively. The nonlinear stiffness (or equivalently the shear stress) is calculated using the distributed element method [15,3], by dividing the shear stressshear strain curve into 500 elastic-perfectly plastic curves, and adding the stress contribution from the individual elastic-perfectly curves to obtain the total shear stress of the soil element. If $G_{i} \bar{\tau}_{y i}$ are the shear modulus and yield stress for the ${ }^{h}$ elastic-perfectly plastic curve,

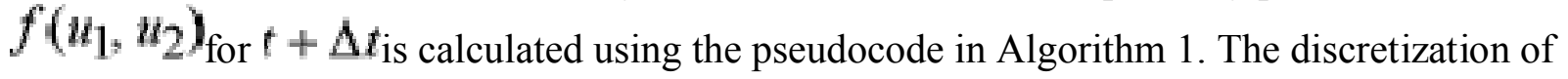
the stress-strain curve into 500 elastic-perfectly plastic curves ensures that numerical error resulting from discretization of stress-strain curve is minimized (solution time history and frequency spectrum of the solution time history up to $100 \mathrm{~Hz}$ converges when $\approx 100$ elasticperfectly plastic curves are considered).

Algorithm 1 Pseudocode to estimate internal force $f\left(u_{1}, u_{2}\right)_{\text {due to nonlinear spring-damper }}$ system at a given time step.

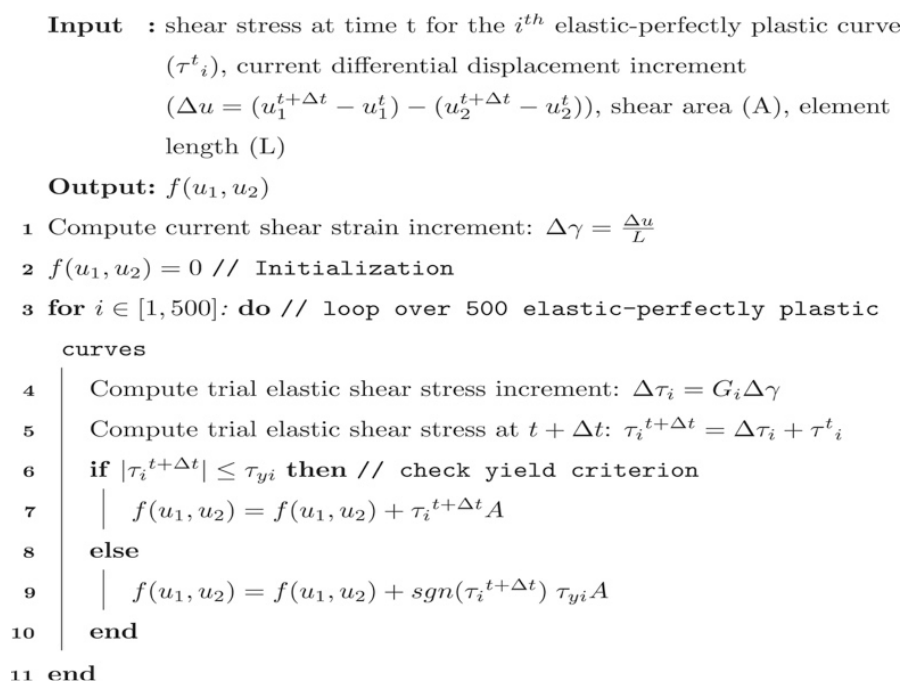

The acceleration time histories at the top (red curve) and base (dashed black curve) of the soil column are presented in Fig. 3b. The acceleration time history at the base is calculated from the applied displacement time history and therefore it is a perfect sinusoidal waveform with a frequency of $1.22 \mathrm{~Hz}$. The acceleration time history at the top of the soil column is not a perfect sinusoidal waveform as can be seen from the peaks and troughs of the red curve in Fig. $3 \mathrm{~b}$. The shear stress time history of the soil element is presented in Fig. 3c. The Fourier transform of the acceleration time history at the top node and of the shear stress time history are presented in Fig. $4 \mathrm{a}$ and $4 \mathrm{~b}$, respectively. The time window for calculating the Fourier transform is chosen such that the maximum amplitude of the input sinusoidal excitation is constant in this window, i.e., from $2 \mathrm{~s}$ to $51 \mathrm{~s}$. These figures show that the acceleration at the top node and the shear stress are made up of sinusoidal waveforms of multiple frequencies even though the input at the bottom node has a single frequency of $1.22 \mathrm{~Hz}$. 

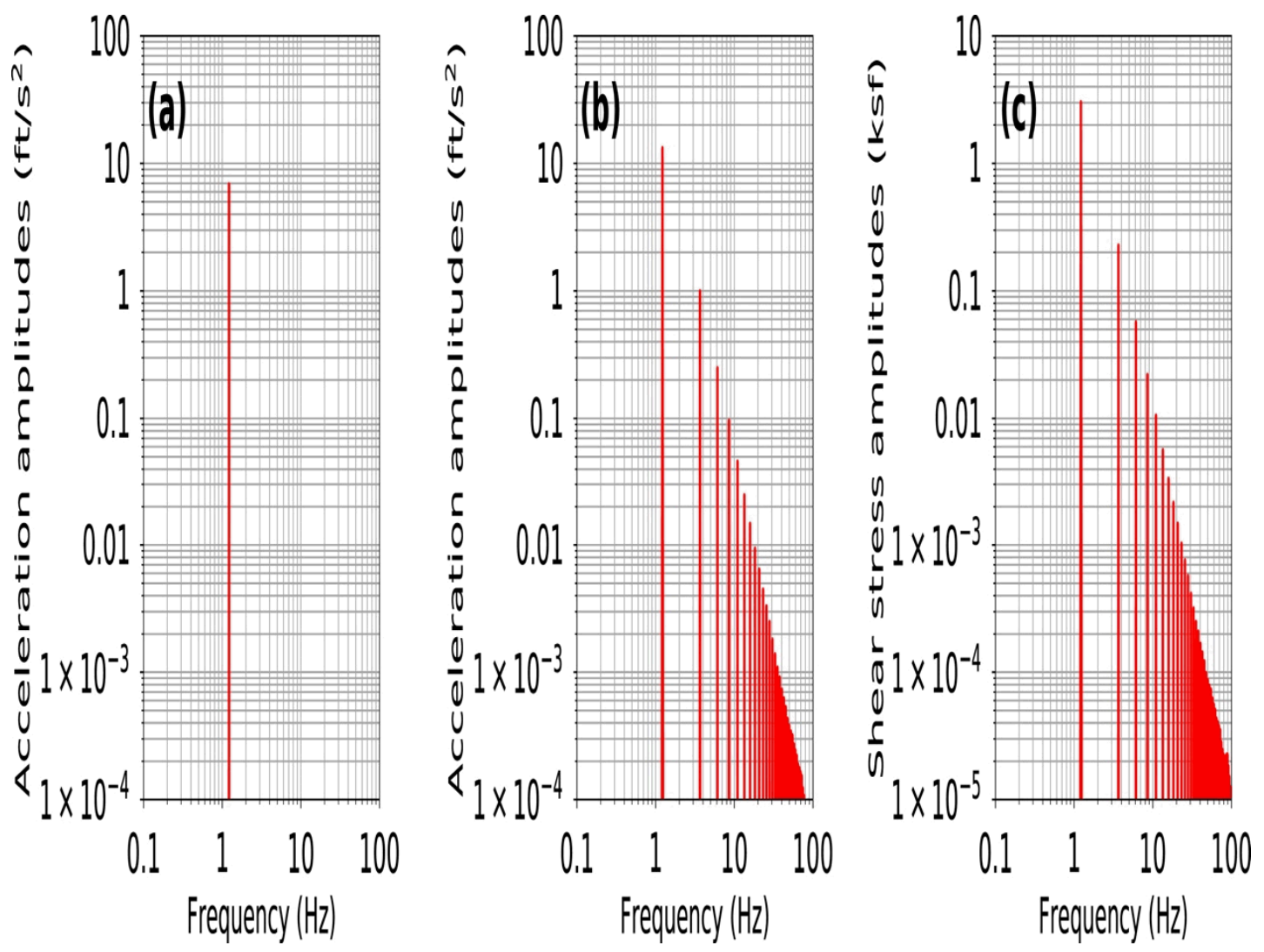

Fig. 4 Fourier amplitude of the (a) input acceleration, (b) acceleration at the top node and (c) shear stress of the soil element. Note that only the section of the time history after the system reaches steady-state, i.e., 2-51 s, is used in calculating the Fourier transform.Fig. 4

A closer look at Fig. 4a shows that majority of the frequency content is still concentrated at $1.22 \mathrm{~Hz}$. Also, the other discrete frequencies present in the Fourier spectrum are at odd multiples or harmonics of the input frequency, i.e., $3.66 \mathrm{~Hz}, 6.10 \mathrm{~Hz}$ and so on. This infinite sine series with odd harmonics is usually seen when a sharp point (or discontinuity in slope) is present in the time history such as in the case of a triangular or square waveform. This is called the Gibbs phenomena [11], where an infinite sum of sine/cosine waveforms, which are smooth functions, are required to represent a piecewise continuously differentiable waveform (i.e., waveforms with a discontinuity in slope). The same trend can also be seen in the Fourier spectrum of the shear stress (Fig. 4b).

The sharp change in slope of the top node's acceleration time history and shear stress time history (Fig. 3b and 3c) can be observed during acceleration reversals or stress reversals, respectively. These reversals correspond to the sharp point in the hysteresis loops, which results in a sudden change in soil stiffness as was identified by Yu et al. [34]. While this is definitely one of the reasons for the presence of frequencies higher than the input frequency, is this the only reason for the high frequency components? Could other aspects of the stress-strain curve, such as the shape of the hysteresis loop in the stress-strain space, result in the higher frequencies? To understand this better, a much simpler problem without the dynamic effects of wave propagation is considered in the next section. Since the Fourier spectrum of the top node's acceleration time 
history and that of the shear stress time history are similar (Fig. 4), we examine only the shear stress time history from this point onwards.

\section{Effect of hysteresis shape}

\subsection{Numerical simulation}

To better test the effect of different stress-strain curves, the same one-element soil column is considered but with a fixed base and a single frequency sinusoidal displacement applied to the top of the soil column (Fig. 5). Prescribing the displacement at the two ends of the soil column ensures that the inertial effects due to the mass of the soil do not play a role in the shear stress calculation. This is equivalent to a nonlinear spring-damper connecting the top and bottom of the soil element. The differential displacement at the two ends of the soil column results in a shear strain, which in turn results in a shear stress. This problem setup helps isolate the effects of the soil's stress-strain curve and is similar to the experimental direct shear test conducted to obtain the stress-strain behavior of the soil. Note that the input sinusoidal displacement considered here (Fig. 5) does not have gradually increasing maximum amplitude as in Fig. 2.
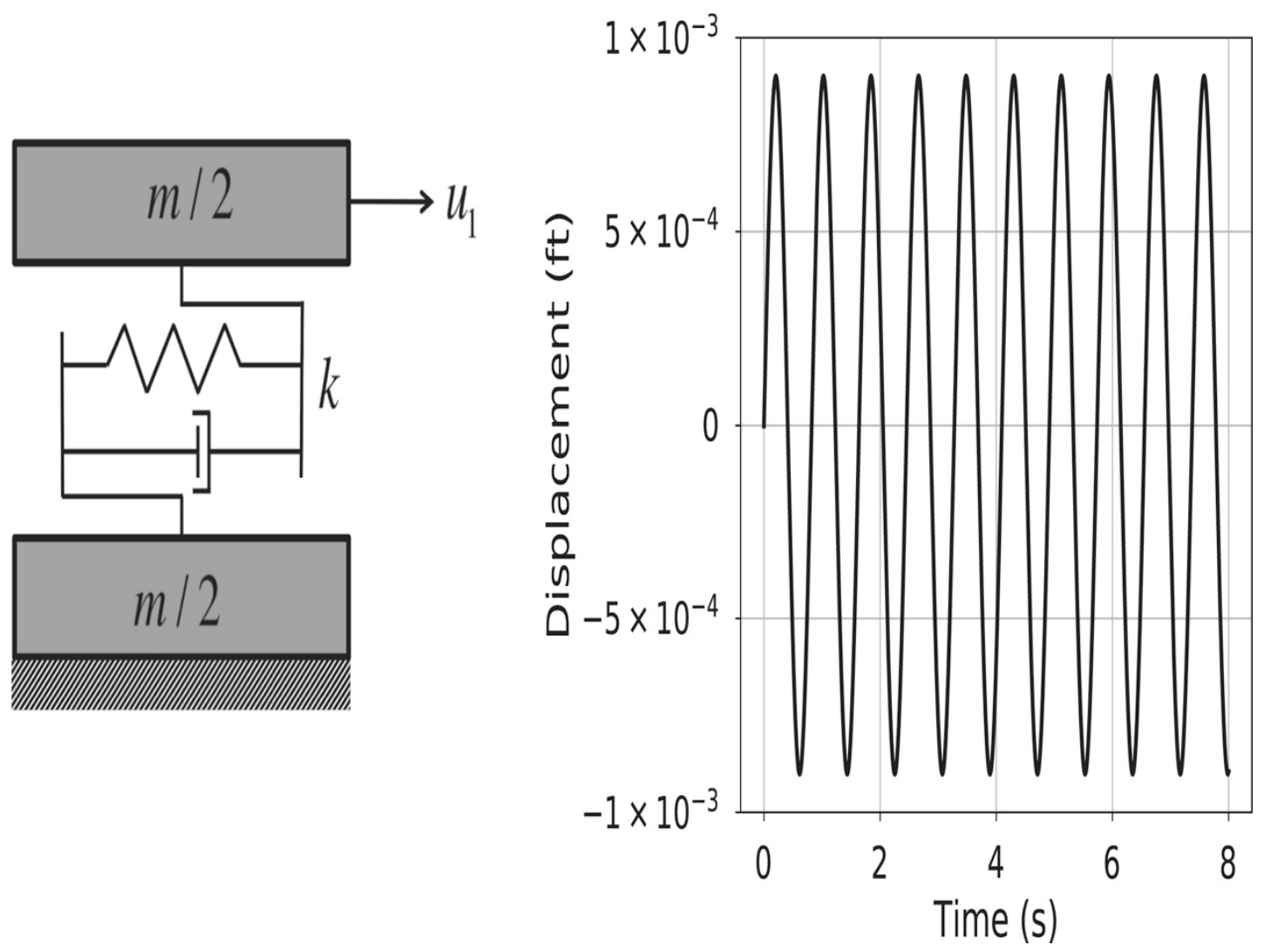

Fig. 5 One-element soil column with fixed base and sinusoidal displacement of frequency $1.22 \mathrm{~Hz}$ applied at the top. This soil element can be compared to that of a nonlinear shear springdamper connecting the top and bottom of the soil column.Fig. 5 
Three different backbone curves are considered for this study. The backbone curves and the resulting hysteresis loops are shown in Fig. 6. The first backbone curve (Fig. 6a) is similar to the stress-strain behavior of alluvial soil and it results in a pointed hysteresis loop as shown in Fig. 6d. The second backbone curve (Fig. 6b) is a modification of the first backbone curve, which results in an elliptical hysteresis loop (Fig. 6e) without any sharp stress reversals. The third backbone curve (red line in Fig. 6c) is obtained by adding a sinusoidal waveform to the central region of the elliptical backbone curve (dashed black line in Fig. 6c). This third backbone curve is designed such that it smoothly converges to the elliptical backbone curve near the ends, i.e., near zero and maximum strain. The addition of the smooth sinusoidal waveform ensures that the hysteresis loop in Fig. $6 \mathrm{f}$ is not a perfect ellipse, but at the same time there are no sharp stress reversals (i.e., the slope of the stress-strain curve is continuous) as in Fig. 6d.
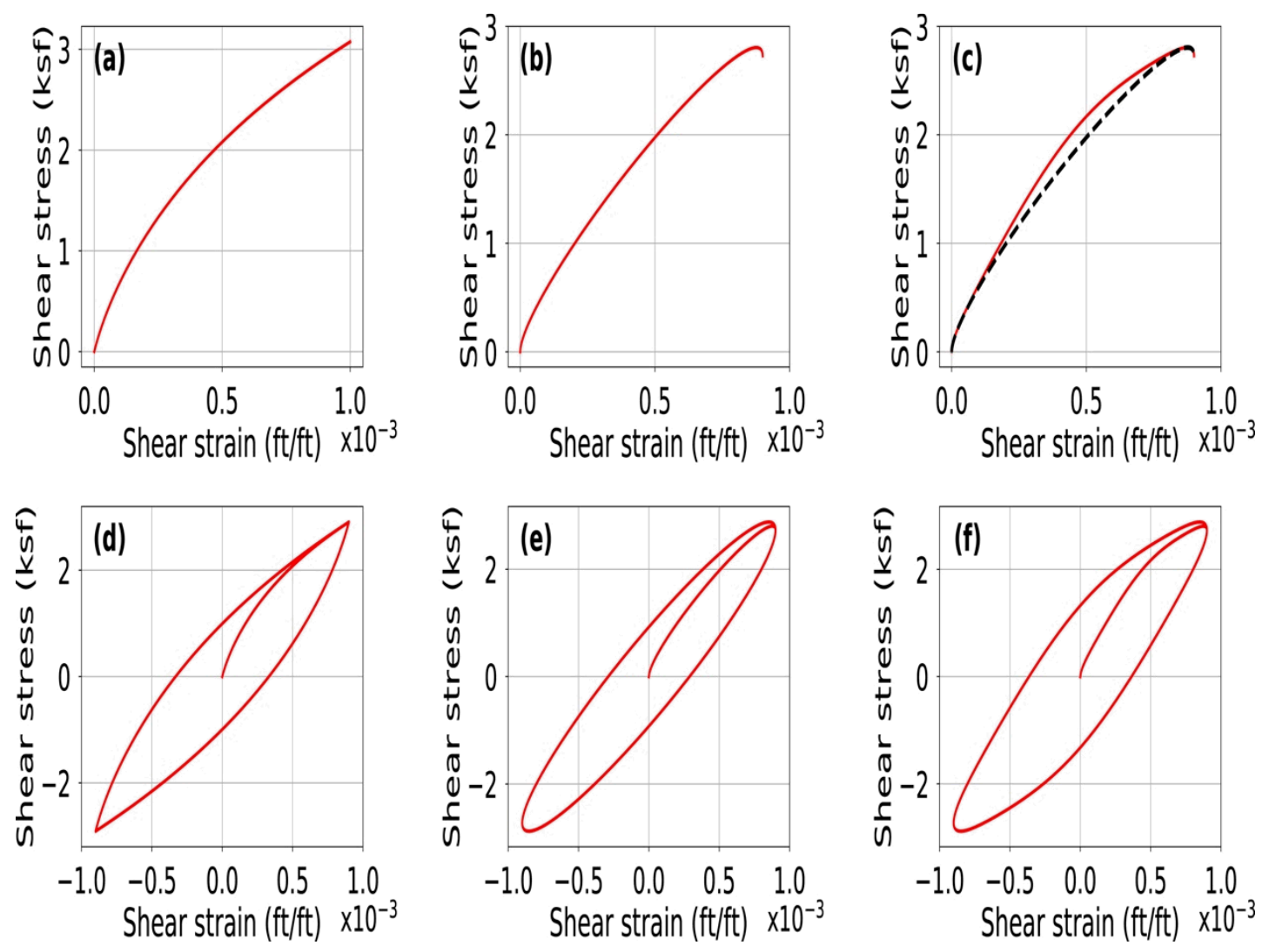

Fig. 6 (a) Alluvial soil, (b) elliptical and (c) smooth pseudo-elliptical backbone curve and the resulting (d) pointed, (d) elliptical and (e) smooth pseudo-elliptical hysteresis loops obtained using Masing hysteresis rules. The backbone curve in (c) (red) is obtained by adding a sinusoidal perturbation to the elliptical backbone curve (dashed black line) from (b).Fig. 6

The shear stress time history for each case is calculated using the distributed element method described in the previous section. The Fourier spectra of the steady-state shear stress time history , i.e, after the first $\mathrm{L} / 4^{t h}$ sinusoidal displacement cycle or $0.205 \mathrm{~s}$, for the three cases are presented in Fig. 7. In the case of the alluvial soil, the pointed hysteresis loop with sharp stress reversals results in the presence of odd multiples of the input frequency (extending to infinity) in the shear stress Fourier spectrum (Fig. 7a). In the case of the elliptical hysteresis loop, the shear 
stress Fourier spectrum (Fig. 7b) contains only one frequency, which is the frequency of the input. This seems to suggest that the pointed nature of the hysteresis loop is the reason for the higher frequencies. But frequencies higher than the input frequency are also present in Fig. 7c, which corresponds to the shear stress Fourier spectrum for the smooth pseudo-elliptical hysteresis loop. In particular, a finite set of odd multiples of the input frequency is present in Fig. 7c as opposed to the infinite set of frequencies in Fig. 7a. This suggests that any deviation of the stress-strain hysteresis shape from a perfect ellipse results in frequencies higher than the input frequency.
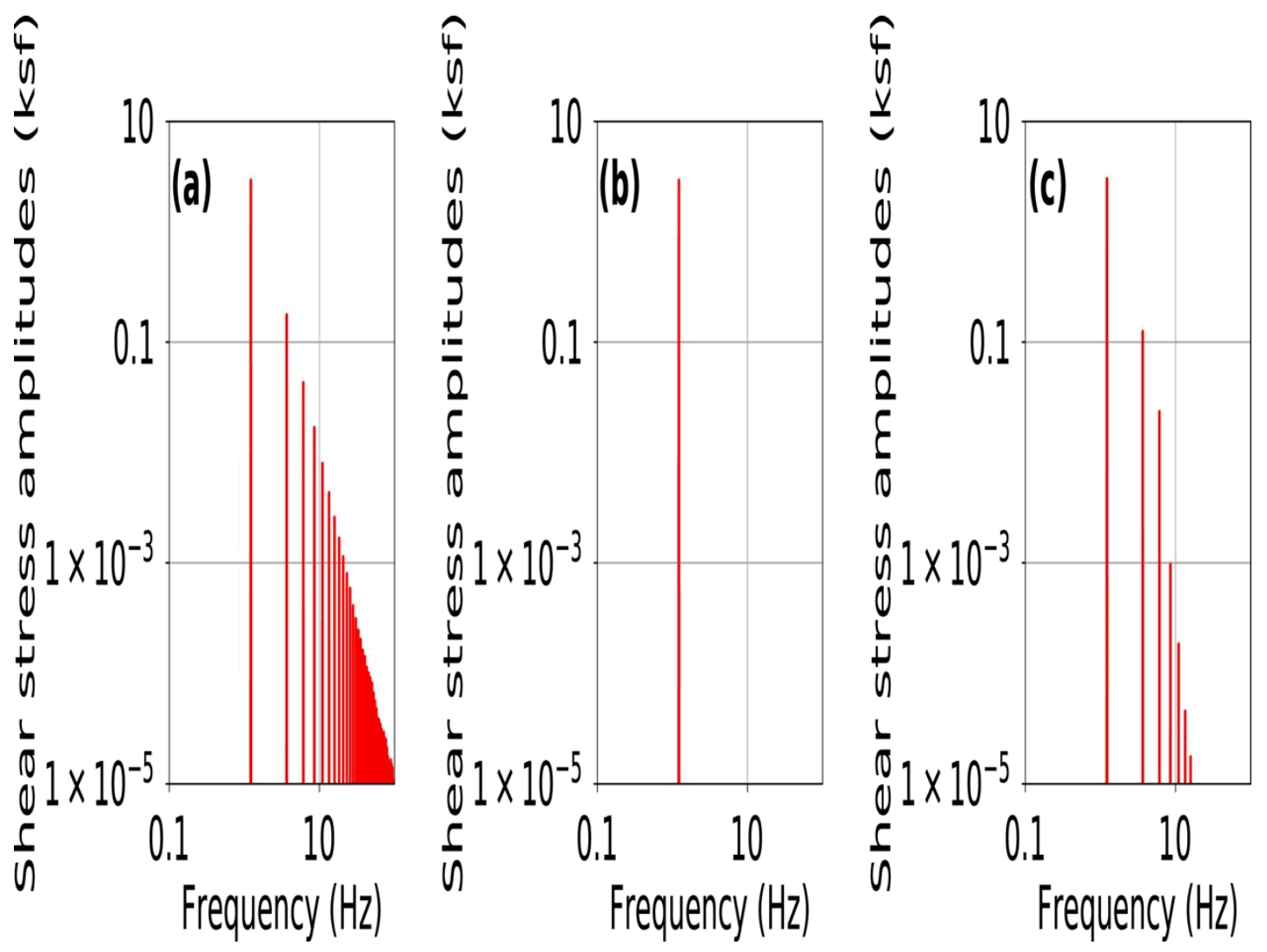

Fig. 7 The Fourier spectrum of the steady-state shear stress time history for (a) alluvial soil, (b) elliptical and (c) smooth pseudo-elliptical backbone curves.Fig. 7

\subsection{Analytical solution}

To understand if the elliptical hysteresis shape is the only shape that results in a single frequency shear stress output for a single frequency displacement input, the equation for the shear stress in terms of shear strain is examined. The stress-strain curve in Fig. 6 is essentially a relation between the shear stress $(\tau)$ and shear strain $(Y)$. Therefore, if the shear stress can be written as a function of shear strain, i.e., $\bar{E}=\boldsymbol{f}(\gamma)$, then that equation describes the shape of the stress-strain hysteresis loop. 
Let the sinusoidal displacement applied at the top node be ${ }^{u_{1}} \equiv A \sin (\omega t)$, where $A$ is the maximum amplitude and $\omega_{\text {is }}$ the frequency of the sinusoidal waveform. The bottom node is fixed implying that the displacement of the bottom node is zero. If $h$ is the height of the soil element, then the resulting shear strain is given by:

$\gamma=\frac{A}{h} \sin (\omega t)$

If the shear stress time history has the same frequency as the input displacement, then $\tau$ can be written as a linear combination of the sine and cosine waveforms with frequency $\omega$ :

$\tau=B \sin (\omega t)+C \cos (\omega t) \equiv B \sin (\omega t)+C \sqrt{1-\sin ^{2}(\omega t)}$

Substituting for $\sin (\omega t)_{\text {from Eq. (2) into Eq. (3) gives: }}$

$$
\begin{aligned}
& \tau=\frac{B h}{A} \gamma+C \sqrt{1-\frac{h^{2}}{A^{2}} \gamma^{2}} \\
& \Rightarrow(A \tau-B h \nu)^{2}=C^{2} A^{2}-C^{2} h^{2} \gamma^{2} \\
& \Rightarrow A^{2} \tau^{2}+\left(B^{2} h^{2}+C^{2} h^{2}\right) \gamma^{2}-2 A B h \tau-C^{2} A^{2}=0
\end{aligned}
$$

The above equation (Eq. (4)), which is a relation between shear stress and shear strain, is the equation for the hysteresis loop in the stress-strain space. This equation looks similar to the equation describing a general conic section, which consists of circle, ellipse, parabola and hyperbola. Eq. (4) is the equation of an ellipse if and only if the square of the coefficient of $T\rangle_{\text {is }}$ less than four times the product of coefficients of $\tau^{2}$ and $\gamma^{2}$, i.e., $(2 A B h)^{2}-4 A^{2}\left(B^{2} h^{2}+C^{2} h^{2}\right)=-4 A^{2} C^{2} h^{2}$ must be less than zero. Since $A^{2}, C^{2}$ and $h^{2}$ are positive, $-4 A^{2} C^{2} h^{2}$ is negative, implying that Eq. (4) is indeed the equation of an

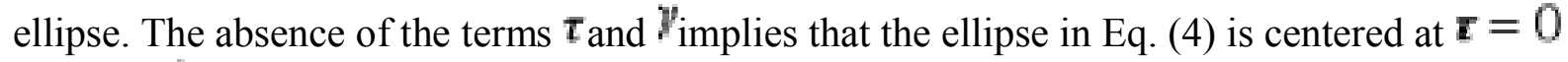
and $\gamma \equiv 0$, and the presence of the cross term $\Gamma$ implies that the major axis of the ellipse is not

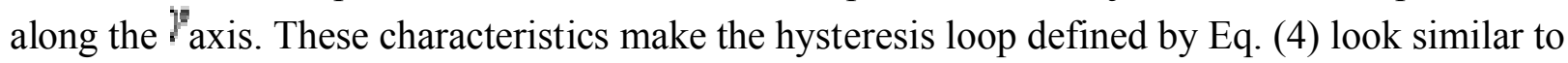
the elliptical hysteresis loop in Fig. 6e.

This proves that if the shear stress contains a single frequency when a single frequency sinusoidal displacement is provided as input to the soil element, then the corresponding shear stress-shear strain curve has to be an ellipse. For all other hysteresis shapes, the shear stress would have frequencies higher than the input frequency. 


\section{Conclusion}

In this article, detailed investigations of nonlinear site response analysis are conducted to understand all the possible causes for the presence of high frequency response in numerical analysis. As a first step, the presence of these high frequency components is demonstrated using a single element soil column that is subjected to a single frequency sinusoidal displacement. Time, soil element and nonlinear stress-strain curve discretization have been handled carefully to minimize numerical errors. This analysis reinforces the conclusions from $\mathrm{Yu}$ et al. [34] that for soils with pointed hysteresis shapes in the stress-strain space, one of the causes for high frequency content in the site response is the sharp stress reversals or the pointed nature of the hysteresis loop.

To understand the other causes for the presence of higher frequencies, a quasi-static problem similar to the direct shear test with displacements prescribed at both ends of the soil column is considered. In this problem, the direct effect of the hysteresis shape is probed by considering three different hysteresis shapes - pointed hysteresis loop, elliptical hysteresis loop and smooth pseudo-elliptical hysteresis loop. The numerical and the analytical solutions presented above show that apart from the sharp stress reversal, any deviation of the hysteresis shape from a perfect ellipse also introduces frequencies higher than the input frequency in the shear stress. While the pointed hysteresis loop results in the presence of odd multiples of the input frequency extending to infinity, any deviation from the elliptical shape, but with a smooth stress reversal, introduces a finite number of odd multiples of the input frequency.

The results presented in this article prove that high frequency components in nonlinear analysis are directly connected to the shape of the hysteresis loops in the stress-strain space. If pointed hysteresis loops or non-elliptical hysteresis loops are a reality, then so are the high frequency components.

\section{Acknowledgements}

This work was sponsored in part by Department of Energy's National Nuclear Security Administration (DOE/NNSAcontract number 102710) and TerraPower through a cooperative research and development agreement (contract number 14-CR-04).

\section{References}

[1]D. Assimaki E. Kausal A. Whittle Model for dynamic shear modulus and damping for granular soils ASCE J Geotech Geoenviron Eng 1262000859869

[2]C. Bolisetti A.S. Whittaker H.B. Mason I. Almufti M. Willford Equivalent linear and nonlinear site response analysis for design and risk assessment of safety related nuclear structures Nucl Eng Des 2742014107121 
[3]D.Y. Chiang J.L. Beck A new class of distributed-element models for cyclic plasticity - i. Theory and application Int J Solids Struct 3141994469484

[4]B.H. Chin L. Aki Simultaneous study of the source, path, and site effects on strong ground motion during the Loma Prieta earthquake: a preliminary result on pervasive nonlinear site effects Bull Seismol Soc Am 81199118591884

[5]J. Coleman C. Bolisetti A. Whittaker Time-domain soil-structure interaction analysis of nuclear facilities Nucl Eng Des 2982016264270

[6]J. Coleman A. Slaughter S. Veeraraghavan C. Bolisetti R. Spears W. Hoffman et al. Mastodon theory manual 2017 Idaho National Laboratory Idaho Falls, Idaho [INL/EXT-17-41930]

[7]Y.F. Dafalias E.P. Popov A model for nonlinearly hardening materials for complex loading Acta Mech 2131979173192

[8]M. Darendeli Development of a new family of normalized modulus reduction and material damping curves [Ph.D. Dissertation] 2001 Department of Civil Engineering, University of Texas Austin

[9]Darragh RB, Shakal AF. The site response of two rock and soil station pairs to strong and weak ground motion. In Proceedings of the 4th international conference on Seismic Zonation, volume 3, pages 359-366, 1991a.

[10]R.B. Darragh A.F. Shakal The site response of two rock and soil station pairs to strong and weak ground motion Bull Seismol Soc Am 81199118851899

[11]J.W. Gibbs Fourier's series Nature 5915221898

[12]D. Groholski Y. Hashash B. Kim M. Musgrove J. Harmon J. Stewart Simplified model for small-strain nonlinearity and strength in $1 \mathrm{~d}$ seismic site response analysis J Geotech Geoenviron Eng 14292016

[13]Y.M.A. Hashash D.R. Groholski C.A. Phillips D. Park M. Musgrove Deepsoil 5.0 user manual and tutorial 2011107

[14]I.M. Idriss H.B. Seed Response of horizontal soil layers during earthquakes 1967 Soil Mechanics and Bituminous Materials Research Laboratory, University of California Berkeley Berkeley, CA

[15]W.D. Iwan On a class of models for the yielding behavior of continuous and composite systems J Appl Mech Trans 34 E3 1967612617

[16]M.K.W. Lee W. Finn DESRA-2: dynamic Effective Stress Response Analysis of soil deposits with energy transmitting boundary including assessment of liquefaction potential 1978 
The University of British Columbia, 335 Faculty of Applied Science Vancouver, British Columbia

[17]LSTC LS DYNA keyword user's manual - release 971 r4 2009 Livermore Software Technology Corporation Livermore, California

[18]W.B. Joyner M.D. Boore L. Seekins Peak acceleration from the 17 October 1989 Loma Prieta earthquake Seismol Res Lett 601989151166

[19]Masing G. Eigenspannungen and verfestigung beim messing. In Proceedings of the 2nd International Congress on Applied Mechanics, Zurich, Switzerland, pp. 332-335, 1926.

[20]N. Matasovic Seismic response of composite horizontally layered soil deposits [Ph.D. Dissertation] 1993 University of California, Los Angeles Los Angeles, CA

[21]N. Matasovic D-MOD 2: a computer program for seismic response analysis of horizontally layered soil deposits, earthfill dams and solid waste landfills 2006 Geomotion, LLC Lacey, WA

[22]Z. Mroz On the description of anisotropic work hardening J Mech Phys Solids 1531967 163175

[23]J.H. Prevost Mathematical modeling of monotonic and cyclic undrained clay behavior Int $\mathrm{J}$ Numer Anal Methods Geomech 121977195216

[24]R.M. Pyke TESS user's manual 2000 TAGA Engineering Software Services Lafayette, CA

[25]W. Ramberg W. Osgood Description of stress-strain curves by three parameters 1943

National Advisory Committee of Aeronautics Washington, DC [Technical Report 902]

[26]K.H. Roscoe J.B. Burland On the generalized stress-strain behavior of wet clay Engineering plasticity 1968 University Press Cambridge 535609

[27]Roscoe KH, Schofield AN. Mechanical behavior of an idealized 'wet' clay. In Proceedings of the 2nd European Conference on Soil Mechanics, Wiesbaden, Germany, volume 1, pages 4754, 1963.

[28]P.B. Schnabel J. Lysmer H.B. Seed Shake: a computer program for earth- quake ground response analysis for horizontally layered sites 1972 Earthquake Engineering Research Center, University of California Berkeley Berkeley, CA [EERC 72-12]

[29]H.B. Seed I.M. Idriss Soil moduli and damping factors for dynamic response analysis 1970 Earthquake Engineering Research Center, University of California Berkeley [EERC 70-10]

[30]J.P. Stewart A. Kwok Y.M.A. Hashash N. Matasovic R. Pyke Z. Wang et al. Benchmarking of nonlinear geotechnical ground response analysis procedure 2008 Pacific Earthquake Engineering Research Center, University of California Berkeley, CA [report 2008/04] 
[31]Dassault Systemes Computer program ABAQUS - finite element analysis software 2005 Dassault Systemes Providence, Rhode Island

[32]R. von Mises Mechanik der festen körper im plastisch deformablen zustand. göttin. nachr Math Phy 11913582592

[33]N. Yoshida S. Kobayashi I. Suetomi K. Muira Equivalent linear method considering frequency dependent characteristics of stiffness and damping Soil Dyn Earthq Eng 222002 205222

[34]G. Yu J.G. Anderson R. Siddarthan On the characteristics of nonlinear soil response Bull Seismol Soc Am 8311992218244 